\section{Estimación etárea del comportamiento sutural en cráneos peruanos precolombinos con deformación cefálica artificial}

\author{
Estimated age of the Sutural behavior in pre-Columbian Peruvian skulls \\ with Artificial cephalic deformation
}

\begin{abstract}
Resumen
Objetivo: Estimar la edad de los cráneos con deformación cefálica artificial a través de la obliteración de las suturas craneanas, palatinas y otros indicadores etaria presentes en la cronología de erupción dentaria; estableciendo relación coherente o discrepante entre ellos. Materiales y método: consistió en 18 cráneos precolombinos comprendidos en la clasificación tipológica de Pedro Weiss.La estimación de la edad está fundamentada en la osificación ectocraneal de las suturas del sistema de la caja craneal y lateral anterior de Meindl \& Lovejoy, en la obliteración de suturas palatinas por el método de Mann, y en otros indicadores etarios; registrándose los grados de cierre sutural de cada uno de los puntos craneométricos; valorados como: $0=$ sutura abierta; $1=$ obliteración mínima; $2=$ obliteración significativa; $3=$ obliteración completa. La sumatoria de los valores obtenidos según Meindl \& Lovejov o la obliteración de la última sutura palatina según Mann o la erupción cronológica de los dientes constituyen la edad estimada; estableciendo linealmente una relación de coherencia o de discrepancia entre ellos. Resultados: La edad de los cráneos con deformación cefálica artificial estimada por cierre de suturas craneanas según el método de Meindl y Lovejoy ${ }^{19}$ expreso valores altos respecto a los estimados por el método de Mann en suturas palatinas. De modo que los valores etarios obtenidos por los métodos suturales expresan la edad aparente, siendo los craneanos más que los palatinos, probablemente por las influencias ambientales. Las suturas palatinas y la dentición al estar alejados de la acción de los aperos deformadores expresan valores más cercanos al real; sin embrago podrían estar influidos por otros factores (malos hábitos). Conclusiones Los resultados sugieren que los aperos deformadores podrían haber acelerado el proceso de osificación de la bóveda craneal en 5 a 10 años aproximados, incrementándose aún más en $15 \pm 5$ años adicionales si se consideran indicadores cronológicos de erupción dentaria.

Palabras claves: suturas craneanas; suturas palatinas; deformación craneana, antropología cultural peruana.
\end{abstract}

\begin{abstract}
Objective:To estimate the age of the skulls with artificial cephalic deformation through the obliteration of the Palatine, cranial sutures and other age indicators present in the chronology of tooth eruption; establishing coherent or conflicting relationship between them. Materials and method: it consisted of 18 pre-Columbian skulls in the typological classification of Pedro Weiss. Estimation of the age is based on ossification ectocraneal of the sutures of the system box cranial and lateral anterior Meindl \& Lovejoy, the obliteration of Palatine by the Mann method sutures, and other indicators of age; registering grades of closure suture of each craneometricos points; rated as: $0=$ open suture; 1 = minimum obliteration; 2 = significant obliteration; 3 = complete obliteration. The sum of the values obtained according to Meindl \& Lovejov or the obliteration of the last midpalatal suture according to Mann or chronological eruption of teeth are the estimated age; establishing a relationship of consistency or discrepancy between them using the straight-line method. Results: the age of the skulls with artificial cephalic deformation estimated by closure of cranial sutures Meindl and express Lovejoy18 method values high on estimates by the method of Mann in palatal sutures. So the age obtained by sutural methods values express the apparent age, being the most cranial to the Palatine, probably by environmental influences. Palatal sutures and the dentition to be away from the action of the deforming Tools Express closer to the actual values; without however could be influenced by other factors (bad habits). Conclusions The results suggest that to deforming tools could have accelerated the process of ossification of cranial vault in 5 to 10 years approximate, further increasing in $15 \pm 5$ additional years if considered chronological indicators of tooth eruption.
\end{abstract}

Key words: cranial sutures; Palatal sutures; cranial deformation, Peruvian cultural.
Artículo Original

\author{
Luis Hernando Gálvez-Calla'; Carlos \\ Alberto Suarez Canlla²; Luis Maita Veliz²; \\ Arnaldo Munive Degregori3; Victoria De \\ Los Ángeles Ramos Arteaga ${ }^{4}$
}

1. Departamento de Ciencias Básicas de la Facultad de Odontología de la Universidad Nacional Mayor de San Marcos. Perú.

2. Departamento Médico Quirúrgico de la Facultad de Odontología de la Universidad Nacional Mayor de San Marcos. Perú.

3. Departamento de Estomatología Rehabilitadora de la Facultad de Odontología de la Universidad Nacional Mayor de San Marcos. Perú.

4. Alumna del Pregrado de la Facultad de Odontología de la Universidad Nacional Mayor de San Marcos. Perú

Correspondencia:

Luis H. Gálvez Calla

Correo electrónico:

lugalca@outlook.com;

lgalvezc@unmsm.edu.pe

Facultad de Odontología, UNMSM. Av. Germán Amezaga 375 Lima 1, Perú

Coautores

Suarez Canlla:

carsuacan@gmail.com

Maita Veliz :

luismv@yahoo.com

Ramos Perfecto:

angeles20ramos@gmail.com

Munive Degregori:

arnaldomunive@hotmail.com

Fecha de recepción: $12 / 5 / 15$

Fecha de aceptación: 10/7/16

() Los autores. Este artículo es publicado por la Revista Odontología Sanmarquina de la Facultad de Odontología, Universidad Nacional Mayor de San Marcos. Este es un artículo de acceso abierto, distribuido 


\section{Introducción}

Los estudios de J. Imbelloni ${ }^{1}$, T. D. Stewart ${ }^{2}$ y P. Weiss ${ }^{3}$ lograron identificar y describir los tipos de deformaciones cefálicas intencionales que se han dado en el continente, su distribución geográfica y sus filiaciones culturales, producidos en el antiguo Perú por llautu y/o por cuna.

Cualquiera que sea el tipo de instrumento deformador este fue aplicado al niño por su propia madre desde el momento de nacer ${ }^{4}$

La bóveda craneana, bajo la presión de los aparatos deformadores, altera su contorno sagital de tal manera que su eje de simetría puede incidir en diversos ángulos sobre el plano aurículo-orbitario o de Frankfurt ${ }^{5}$, determinando formas con tendencias braquicéfalos y dolicocéfalos ${ }^{6}$

La deformación craneana responde a cambios en las presiones sociales y ambientales $^{7}$

El cráneo, al estar formado por una serie de huesos separados, presenta una coordinación entre el crecimiento del mismo y la expansión del cerebro en desarrollo ${ }^{8}$ mediante el movimiento pasivo de los huesos?

Las suturas son los tejidos fibrosos que mantienen unidos los huesos del cráneo ${ }^{10}$ y representan el lugar donde se produce el crecimiento óseo, propiamente en los márgenes adyacentes de los huesos ${ }^{8}$. La fusión ósea (obliteración completa) no ocurre normalmente hasta una edad avanzada en el cráneo humano, a excepción de la sutura metópica que suele fusionarse entre los 18 meses $^{8}$ y los 5 ańos de edad ${ }^{10}$, quedando consolidado alrededor de los 7 años; le siguen las suturas sagital, coronal y lambdoidea entre los 22 y 35 ańos ${ }^{11,12}$

El hueso es un tejido modulado ontogenética y filogenéticamente, por tanto sensible a diferentes factores ${ }^{13}$.

Es indudable que las vendas $y / 0$ tablillas (factores ambientales externos) aplicadas después del nacimiento podrían haber movilizado los diferentes huesos que conforman el cráneo estimulando actividades osetoblásticas y osteoclásticas en regiones fontanelares y suturales, acelerando los procesos osteogénicos y de remodelación en estas regiones con la consiguiente deformación cefálica producto del cierre precoz y obliteración en diversos grados de una o varias suturas comprometidas ${ }^{6}$
La obliteración de las suturas de la bóveda craneal comienza por lo común entre los 30 a 40 ańos por la cara interna y unos 10 ańos más tarde por la externa $^{14}$

En los humanos la sutura metópica se cierra entre el segundo y quinto ańo después del nacimiento, pero en el 10\% de la población la sutura permanece permeable $^{10}$; mientras que, la sutura coronal, sagital y lambdoidea se cierran entre los 22 y los 40 años $^{15,16}$

Sin embargo en la cara los huesos están separados por uniones fibrosas hasta los 70 u 80 años de vida ${ }^{14}$

Las suturas, escamosa, occipitomastoidea y esfenotemporal pueden permanecer abiertas hasta los 70 ańos ${ }^{15}$

La permeabilidad y obliteración de las suturas pueden ser atribuidas a la presencia o ausencia de fuerzas físicas externas (factores ambientales) en los distintos huesos de cráneo, siendo el tejido conjuntivo de la sutura craneal el que se adaptaría totalmente a las demandas funcionales del entorno biomecánico de la sutura ${ }^{17}$

La estimación de la edad representa el procedimiento más complejo del proceso de identificación de restos óseos, especialmente en adultos, pues los fenómenos de envejecimiento varían según la población, el sexo, el ambiente, el estatus social y las condiciones de salud de la persona. En la medida que se incrementa la edad del individuo, aumenta, asimismo, el margen de error ${ }^{18}$.

Meindl y Lovejoy ${ }^{19}$, establecieron indicadores de edad craneal utilizando 7 puntos de la bóveda craneana (midlambdoideo, lambda, obelion, sagital anterior, bregma, midcoronal, pterion), al que se le denomino Sistema de la Caja Craneana (Fig. 5)

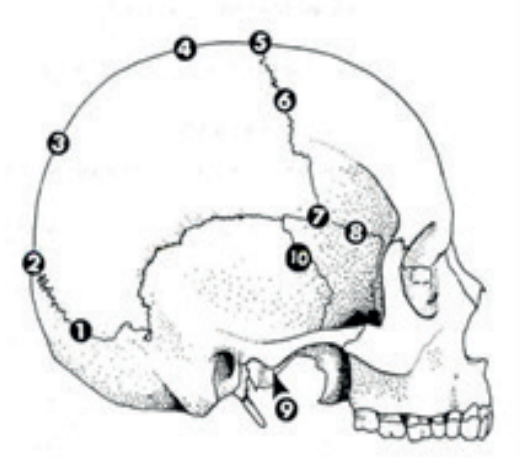

Fig. 5: Puntos ectocraneales para la observación de la obliteración (por Meindl \& Lovejoy 1985)
El sistema lateral anterior (midcoronal, pterion, esfenofrontal, esfenotemporal inferior y el esfenotemporal superior), constituye el mejor indicador de la edad de un craneo $^{19}$

Para lo cual se estableció una escala de valoración del grado de cierre sutural de 0-3, (Figs.:1,2,3,4) seleccionando una región específica de $1 \mathrm{~cm}$ de longitud alrededor del punto craneal a observar, apreciando el grado de cierre sutural a trasluz, prefiriendo la tabla externa (ectocraneal) por cuanto la actividad de obliteración es más rápida a nivel interno (endocraneal) ${ }^{19}$.

De manera objetiva estableció lo siguiente $^{19}$ :

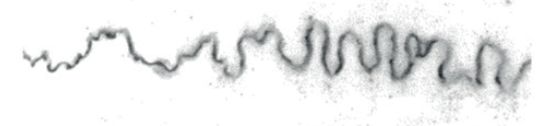

Fig. 1: Grado 0: sutura abierta. Sin evidencia de osificación ectocraneal (tabla externa). Cortesía de P. L. Walker Standards-For Data Collection From Human Skeletal Remains

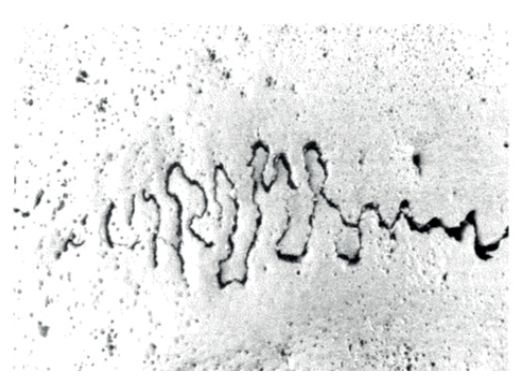

Fig. 2: Grado 1, cierre mínimo, formación de un puente óseo mínima que cruza la sutura que puede alcanzar hasta el $50 \%$ en el sitio de cierre sutural. Cortesía de P. L. Walker Standards-For Data Collection From Human Skeletal Remains

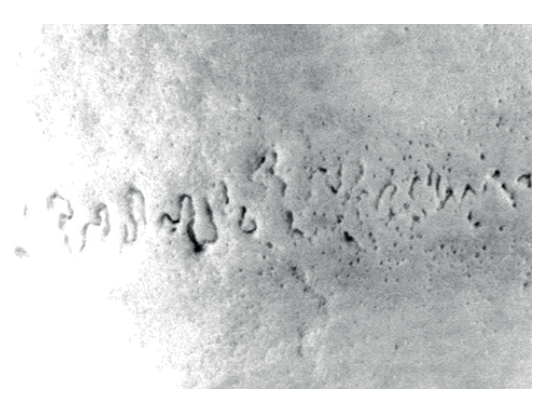

Fig. 3: Grado 2, cierre significativo, marcado grado de cierre que puede alcanzar hasta el $80 \%$ de cierre en el sitio. Cortesía de P. L. Walker Standards-For Data Collection From Human Skeletal Remains 


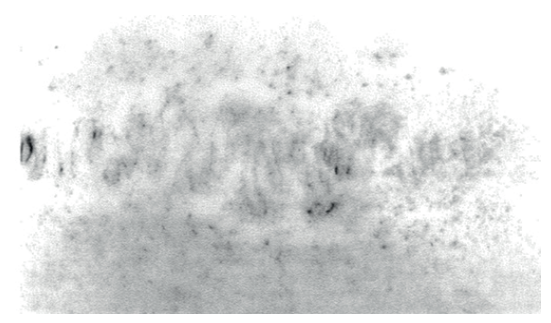

Fig. 4: Grado 3, Obliteración completa, fusión total del sitio. Cortesía de P. L. Walker StandardsFor Data Collection From Human Skeletal Remains

Según los coeficientes de correlación los puntos pterion, esfenofrontal, midlambdoideo y lambdoideo son los que más se relacionan con el incremento de la edad. El punto pterion parece ser el mejor indicador del advenimiento de la década de los 40 años; el esfenotemporal refleja procesos característicos de las edades superiores a esta década; el obelion exhibe una actividad temprana en la mayoría de los casos; el punto esfenofrontal inicia su obliteración después del midcoronal ${ }^{19}$.

En una muestra de cementerio y de morgue de Bogotá integrada por 100 individuos de distintas edades, aunque con sobre representación de edades superiores a 60 años y sub representación de cohortes entre 35-55 años ${ }^{20}$, aplicando el método de Meindl y Lovejoy ${ }^{19}$ se encontró que el sistema de la bóveda craneal es más confiable que el lateral; y los puntos obelion, pterion y esfenofrontal son los que más se relacionan con la edad, es decir, a mayor puntaje mayor edad ${ }^{20}$.

Cuando un espécimen presenta obliteración completa de sus suturas se debe verificar su edad utilizando otros indicadores del esqueleto. El sexo y la filiación poblacional contribuyen sesgos insignificantes en el diagnóstico de la edad.

Mann et al. ${ }^{21}$ sugirió que las cuatro suturas palatinas (incisiva, interpalatina, intermaxilar y palatomaxilar) pueden ser indicadores generales para la estimación de la edad. En su estudio: Obliteración de la sutura maxilar ${ }^{21}$, concluyó que el análisis de la obliteración de estas suturas es útil para estimar la edad biológica general. Posteriormente en otro estudio: Obliteración de la Sutura Maxilar: un método visual para la estimación de edad ósea ${ }^{22}$, concluyó que aun cuando éste método no arroja una estimación exacta de la edad es muy valioso para establecer el rango de edad.

La secuencia de cierre sigue un patrón de conducta regular comenzando por la sutura incisiva, sutura transversa palatina, luego la sutura palatina media pos- terior y finalmente la sutura palatina anterior ${ }^{22}$.

López $\mathrm{PJ}^{23}$ señala que un individuo menor de 18 ańos presenta ninguna o poca obliteración de la sutura incisiva en menos de la mitad y ninguna obliteración de otras suturas maxilares palatinas; un adulto mayor de 18 años presenta alguna obliteración de la sutura interpalatina y la sutura incisiva cerrada en más de la mitad; en un adulto mayor de 50 años, todas las suturas están cerradas. López establece rangos de edad más amplios que los de Mann para evidencia de obliteración de las suturas ${ }^{23}$.

Se estableció en Perú, siguiendo el método de Mann, una secuencia de obliteración de suturas palatinas según edad estimada $^{24}$ : sutura incisiva (20 a 25 años de edad); sutura incisiva y sutura transversal palatina (26 a 32 años); sutura incisiva, transverso palatina y palatina media posterior (33 a 39 años); finalmente, sutura incisiva, transversa palatina, palatina media posterior y sutura palatina media anterior $(40$ a 46 años de edad).

El presente estudio se realizó con la finalidad de estimar la edad de los cráneos precolombinos con deformación cefálica artificial determinada a través de la obliteración de las suturas craneanas, palatinas y otros indicadores presentes en la cronología de erupción dentaria, estableciendo una relación coherente o discrepante entre ellos, siendo los objetivos los siguientes:

- Estimar la edad de los cráneos precolombinos con deformación cefálica artificial determinada a través de la obliteración de las suturas craneanas, palatinas y otros indicadores etarios presentes en la cronología de erupción dentaria.

- Estimar la edad de los cráneos humanos precolombinos con deformación cefálica artificial a través de indicadores etarios presentes en las suturas del sistema de la caja craneal anterior (método de Meindl y Lovejoy) $)^{19}$.

- Estimar la edad de los cráneos humanos precolombinos con deformación cefálica artificial por medio de indicadores etarios presentes en las suturas palatinas (método de Mann) ${ }^{21,22 .}$

- Estimar la edad de los cráneos humanos precolombinos con deformación cefálica artificial por medio de los indicadores etarios presentes en la cronología de erupción dentaria

- Observar discrepancias existentes, en una relación horizontal, entre las edades estimadas mediante los indicadores descritos en los objetivos específicos, estimando la edad real y aparente de los cráneos en cuestión.

\section{Materiales y método}

Consistió en 18 cráneos precolombinos comprendidos en la clasificación tipológica de Pedro Weiss, Se utilizo :

Estimación de la edad por el método de Meindl \& Lovejoy ${ }^{19}$ que consiste en observar las regiones circundantes a los puntos craneométricos del sistema de la caja craneal y del sistema lateral anterior; a $1 \mathrm{~cm}$ adyacente a los puntos craneométricos, apreciando el grado de cierre sutural ectocraneal a través de un zoom óptico de alta resolución. Luego se registraran los grados de cierre sutural en cada uno de los puntos craneométricos de acuerdo a la siguiente valoración: $0=$ abierta, sin evidencia de cierre ectocraneal; $1=$ con cierre mínimo; $2=$ con cierre significativo; $3=$ con obliteración completa. Una vez establecida las valoraciones en zonas adyacentes a cada punto craneométrico del sistema de la caja craneal y del sistema lateral anterior, se procede a la sumatoria de los valores obtenidos que constituye la edad estimada.

Estimación de la edad por el método de Mann, ${ }^{21,22}$ última obliteración de la sutura palatina. Según secuencia de obliteración de suturas palatinas: 1 . Sutura incisiva, 2. Sutura transversal palatina, 3. Sutura palatina posterior y 4 . Sutura palatina anterior; con las mismas valoraciones de grados de cierre sutural.

A efectos de la estimación etaria se consideró la secuencia de obliteración de suturas palatinas según edad sugerida por Navarro \& Roldán. ${ }^{24}$ sutura incisiva (20 a 25 años de edad); sutura incisiva y sutura transversal palatina (26 a 32 ańos); sutura incisiva, transverso palatina y palatina media posterior (33 a 39 años); finalmente, sutura incisiva, transversa palatina, palatina media posterior y sutura palatina media anterior (40 a 46 años de edad).

La estimación de la edad determina$\mathrm{da}$ por indicadores cronológicos de la erupción dentaria tiene sus bases en los establecidos por De Schour y Massier (1941). ${ }^{25}$

\section{Resultados.}

Las edades estimadas de 18 cráneos precolombinos con deformación cefálica artificial, comprendidos en la clasificación tipológica de Pedro Weiss, fueron determinadas según: 
- Obliteración de suturas del Sistema de la Caja Craneal de Meindl \& Lovejoy $^{19}$

Tabla 1: Estimación de la edad de los cráneos con deformación cefálica artificial por obliteración de suturas del Sistema de la Caja Craneal de Meindl \& Lovejoy

\begin{tabular}{|c|c|c|c|c|c|c|c|c|c|}
\hline \multirow{2}{*}{$\begin{array}{c}\mathrm{N}^{\circ} \\
\text { craneos }\end{array}$} & \multirow{2}{*}{\begin{tabular}{|c|} 
Cráneos \\
defor- \\
mados \\
clasifi- \\
cación \\
tipológica \\
de Weiss \\
\end{tabular}} & \multirow{2}{*}{ Código } & \multicolumn{7}{|c|}{$\begin{array}{l}\text { Obliteración sutural } \\
\text { Método Meindl \& Lovejov } \\
\text { Sistema de la Caja Craneal }\end{array}$} \\
\hline & & & 1 & 23 & 4 & 5 & 67 & Total & $\begin{array}{c}\text { Edad } \\
\text { (años) }\end{array}$ \\
\hline \multirow{2}{*}{2} & \multirow{2}{*}{$\begin{array}{c}\text { Fronto } \\
\text { occipital }\end{array}$} & AF4885 & 3 & 35 & 3 & 2 & $2-$ & 16 & 48.8 \\
\hline & & AF6025 & 0 & 0 & 0 & 0 & 02 & 3 & 34.7 \\
\hline 1 & $\begin{array}{c}\text { Inca } \\
\text { costeño }\end{array}$ & AF0767 & 0 & 0 & 1 & & 00 & 2 & 30.5 \\
\hline \multirow{2}{*}{2} & \multirow{2}{*}{ Aimara } & AF10870 & & 0 & $\underline{0}$ & & $\begin{array}{ll}0 & 0 \\
\end{array}$ & 1 & 30.5 \\
\hline & & AF1489 & 0 & 0 & 0 & & 00 & 0 & $<30$ \\
\hline \multirow{2}{*}{2} & \multirow{2}{*}{ Opa } & AF1643 & 1 & 12 & 2 & 0 & 13 & 10 & 39.4 \\
\hline & & AF1577 & - & - & - & & -0 & 0 & $<30$ \\
\hline \multirow{2}{*}{2} & \multirow{2}{*}{ Cavernas } & AF1763 & 0 & 0 & 0 & & 00 & 0 & $<30$ \\
\hline & & AF1741 & 0 & 0 & 0 & & 00 & 0 & $<30$ \\
\hline \multirow[b]{2}{*}{2} & \multirow{2}{*}{ Huaura } & AF4820 & 0 & 0 & 0 & & 00 & 0 & $<30$ \\
\hline & & AF1699 & 0 & 0 & 0 & & 00 & 0 & $<30$ \\
\hline & \multirow{2}{*}{$\begin{array}{c}\text { Cabeza } \\
\text { larga } \\
\end{array}$} & AF2613 & 2 & 35 & & & 11 & 12 & 45.2 \\
\hline & & AF5954 & 0 & 1 1 & 1 & 0 & 12 & 5 & 34.7 \\
\hline \multirow[b]{2}{*}{2} & \multirow{2}{*}{ Necrópolis } & AF6797 & 3 & 25 & 2 & & 23 & 17 & 48.8 \\
\hline & & AF6830 & 0 & 0 & 0 & & 01 & 2 & 30.5 \\
\hline \multirow{2}{*}{2} & \multirow{3}{*}{$\begin{array}{c}\begin{array}{c}\text { Natchez } \\
\text { momia }\end{array} \\
\end{array}$} & AF6823 & 1 & 13 & 3 & 3 & 32 & 16 & 48.8 \\
\hline & & 108 & 0 & 0 & & & & 3 & 34.7 \\
\hline 1 & & AF1648 & \begin{tabular}{|l|l|}
1 \\
\end{tabular} & 0 & 0 & & 12 & 4 & 34.7 \\
\hline
\end{tabular}

Puntos craneométricos: 1. Midlambdoideo, 2. Lambda, 3. Obelión, 4. Sagital anterior, 5. Brergma, 6. Midcoronal, 7. Pterion. Grados de cierre sutural: 0 = sutura abierta, $1=$ cierre mínimo $(50 \%), 2$ = cierre significativo $(80 \%), 3$ = Obliteración Completa (100\%)

- La edad de los cráneos con deformación Fronto-occipital (AF4885), (Fig.6), necrópolis (AF6797) y Natchez (AF6823), (Fig.8) fueron de 48.8 ańos, seguidos de 1 cráneo tipo Cabeza Larga (AF2613) de 45.2 años.

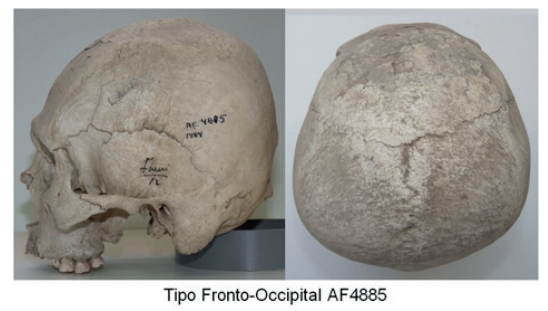

Fig.6: Cráneo precolombino con deformación cefálica tipo Fronto-occipital, se aprecia obliteración sutural grado 3 por norma superior

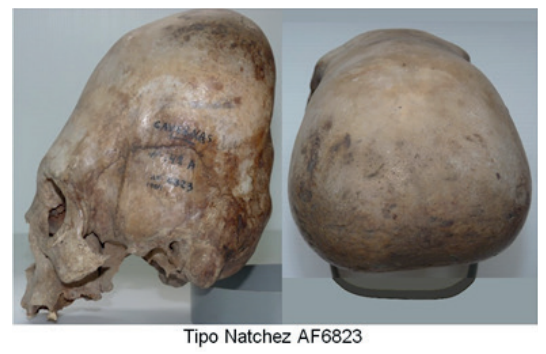

Fig. 8: Cráneo precolombino con deformación cefálica tipo Natchez, se aprecia obliteración sutural grado 3 por norma superior
Un cráneo tipo Opa (AF1643) estimó 39.4 años, seguidos de 1 Frontooccipital (AF6025), 1 Cabeza Larga (AF5954), 1 Natchez (momia 108) y 1 Nazca (AF1648) que estimaron 34.7 años.

Los demás cráneos con deformación cefálica artificial, 1 Inca Costeño (AF0767), 1 Aimara (AF10870), 1 Necrópolis (AF6830) estimaron 30.5 años; por último, los cráneos tipo $\mathrm{Ai}$ mara (AF1489), Opa (AF1577), 2 Cavernas (AF1763, AF1741) y 2 Huaura (AF4820, AF1699) estimaron menos de 30 ańos al momento de su muerte (Fig.10).Obliteración de suturas del Sistema Lateral Anterior de Meindl \& Lovejoy $^{19}$

Tabla 2: Estimación de la edad de los cráneos con deformación cefálica artificial por obliteración de suturas del Sistema Lateral Anterior de Meindl \& Lovejoy

\begin{tabular}{|c|c|c|c|c|c|c|c|c|c|}
\hline \multirow{2}{*}{$\begin{array}{c}\mathrm{N}^{\circ} \\
\text { craneos }\end{array}$} & \multirow{2}{*}{$\begin{array}{c}\text { Cráneos } \\
\text { deforma- } \\
\text { dos cla- } \\
\text { sificación } \\
\text { tipológica } \\
\text { de Weiss }\end{array}$} & \multirow[b]{2}{*}{ Código } & \multicolumn{7}{|c|}{$\begin{array}{l}\text { Obliteración sutural } \\
\text { Método Meindl \& Lovejov } \\
\text { Sistema Lateral Anterior }\end{array}$} \\
\hline & & & 6 & 7 & 8 & 9 & 10 & Total & $\begin{array}{l}\text { Edad } \\
\text { (años) }\end{array}$ \\
\hline \multirow{2}{*}{2} & \multirow{2}{*}{$\begin{array}{c}\text { Fronto } \\
\text { occipital }\end{array}$} & AF4885 & 2 & - & & 1 & - & & - \\
\hline & & AF6025 & 0 & 2 & 0 & 0 & 0 & 2 & 36.2 \\
\hline 1 & $\begin{array}{c}\text { Inca } \\
\text { costeño }\end{array}$ & AF0767 & 0 & 0 & 0 & 1 & 0 & 1 & 32 \\
\hline \multirow{2}{*}{2} & \multirow{2}{*}{ Aimara } & AF10870 & 0 & 0 & 2 & 0 & 0 & 2 & 36.2 \\
\hline & & AF1489 & 0 & 0 & 0 & 0 & 0 & 0 & $<30$ \\
\hline \multirow{2}{*}{2} & \multirow{2}{*}{ Opa } & AF1643 & 1 & 3 & 3 & 2 & 0 & 9 & 51.9 \\
\hline & & AF1577 & - & 0 & 0 & 0 & 0 & & - \\
\hline \multirow{2}{*}{2} & \multirow{2}{*}{ Cavernas } & AF1763 & 0 & 0 & 1 & 0 & 0 & 1 & 32 \\
\hline & & AF1741 & 0 & 0 & 1 & 0 & 0 & 1 & 32 \\
\hline \multirow[b]{2}{*}{2} & \multirow{2}{*}{ Huaura } & AF4820 & 0 & 0 & 0 & 0 & 0 & 0 & $<30$ \\
\hline & & AF1699 & 0 & 0 & 0 & 1 & 0 & 1 & 32 \\
\hline \multirow{2}{*}{2} & \multirow{2}{*}{$\begin{array}{c}\text { Cabeza } \\
\text { larga }\end{array}$} & AF2613 & 1 & \begin{tabular}{|l}
1 \\
\end{tabular} & 2 & 3 & 0 & 7 & 45.5 \\
\hline & & AF5954 & 1 & 2 & 1 & 0 & 1 & 5 & 41.1 \\
\hline \multirow[b]{2}{*}{2} & \multirow{2}{*}{ Necrópolis } & AF6797 & 2 & 3 & 3 & 2 & 3 & 13 & 56.2 \\
\hline & & $\begin{array}{l}\text { AF6830 } \\
\end{array}$ & $\frac{2}{0}$ & \begin{tabular}{|l|}
1 \\
\end{tabular} & 0 & \begin{tabular}{|l} 
\\
0
\end{tabular} & 0 & 1 & $\frac{0.2}{32}$ \\
\hline \multirow[b]{2}{*}{2} & \multirow{3}{*}{$\begin{array}{l}\text { Natchez } \\
\text { momia }\end{array}$} & AF6823 & 3 & 2 & 0 & 0 & 0 & 5 & 41.1 \\
\hline & & 108 & 1 & 2 & 2 & 0 & 0 & 5 & 41.1 \\
\hline 1 & & AF1648 & 1 & 2 & 2 & 0 & 0 & 5 & 41.1 \\
\hline
\end{tabular}

Puntos craneométricos: (6) Midcoronal, (7) Pterion, (8) Esfenofrontal, (9) Esfenotemporal inferior, (10) Esfenotemporal superior. Grados de cierre sutural: $0=$ sutura abierta, $1=$ cierre mínimo (50\%), 2 = cierre significativo $(80 \%), 3$ = Obliteración Completa (100\%)

Uno de los cráneos con deformación Fronto-occipital (AF6025) tuvo 36.2 ańos y el otro (AF4885) debido a que los indicadores fueron alterados e imposibilitaron su registro se tomó en cuenta en este caso el sistema de la Caja Craneal estableciéndose la edad de 48.8 años. Del mismo modo la edad determinada del cráneo con deformación Inca Costeño (AF0767) fue de 32 años.

Respecto a los cráneos tipo Aimara, uno de ellos (AF10870) tuvo 36.2 ańos de edad; y el otro (AF1489) menos de 30 ańos. Igualmente, la edad de un cráneo con deformación Opa (AF1643) fue de 51.9 años y la del otro (AF1577) de menos 30 años.

Las edades de los cráneos con deformación tipo Cavernas (AF1763 y AF1741) fueron de 32 años. Del mismo modo, la edad de uno de los cráneos tipo Huaura (AF1699) fue de 32 ańos y del otro (AF4820) fue de menos de 30 años.

Las edades de los cráneos tipo Cabeza Larga fueron de 45.5 (AF2613) y 41.1 (AF5954) años. De igual manera, las edades de los cráneos con deformación tipo Necrópolis fueron de 56.2 (AF6797) años y de 32 (AF6830) años. Finalmente, las edades de los cráneos tipo Natchez (AF6823 y Momia 108) y Nazca (AF1648) fueron de 41.1 años.

- Obliteración de Suturas Palatinas de Mann ${ }^{21,22}$

Tabla 3: Estimación de la edad de los cráneos con deformación cefálica artificial por obliteración de suturas palatinas de Mann

\begin{tabular}{|c|c|c|c|c|c|c|}
\hline \multirow[t]{2}{*}{$\begin{array}{l}\mathrm{N}^{\circ} \\
\text { craneos }\end{array}$} & \multirow{2}{*}{$\begin{array}{l}\text { Cráneos deforma- } \\
\text { dos clasificación } \\
\text { tipológica de } \\
\text { Weiss }\end{array}$} & \multirow[t]{2}{*}{ Código } & \multicolumn{4}{|c|}{$\begin{array}{l}\text { Obliteración sutural } \\
\text { Método Mann } \\
\text { Suturas de la Bóveda } \\
\text { Palatina }\end{array}$} \\
\hline & & & 1 & 2 & \begin{tabular}{l|l}
3 & 4
\end{tabular} & Edad (años) \\
\hline \multirow{2}{*}{2} & \multirow{2}{*}{ Fronto occipital } & AF4885 & 3 & 2 & \begin{tabular}{l|l}
2 & 0
\end{tabular} & $33-39$ \\
\hline & & AF6025 & 2 & - & -0 & $18-20$ \\
\hline 1 & Inca costeño & AF0767 & 3 & 1 & $\begin{array}{lll}0 & 0 \\
\end{array}$ & $26-30$ \\
\hline \multirow{2}{*}{2} & \multirow{2}{*}{ Aimara } & AF10870 & 3 & 1 & \begin{tabular}{l|l}
1 & 0 \\
\end{tabular} & $29-33$ \\
\hline & & AF1489 & - & 0 & -- & $<18$ \\
\hline \multirow{2}{*}{2} & \multirow{2}{*}{ Opa } & AF1643 & 3 & 2 & \begin{tabular}{|l|l|}
2 & 1 \\
\end{tabular} & $36-40$ \\
\hline & & AF1577 & 1 & 1 & 10 & $20-33$ \\
\hline \multirow{2}{*}{2} & \multirow{2}{*}{ Cavernas } & AF1763 & 3 & 2 & \begin{tabular}{|l|l|}
2 & 1 \\
\end{tabular} & $30-40$ \\
\hline & & AF1741 & 1 & 1 & \begin{tabular}{l|l}
0 & 0
\end{tabular} & $20-26$ \\
\hline \multirow{2}{*}{2} & \multirow{2}{*}{ Huaura } & AF4820 & 1 & 1 & 10 & $20-33$ \\
\hline & & AF1699 & 3 & 1 & $\begin{array}{ll}0 & 0 \\
\end{array}$ & $26-30$ \\
\hline \multirow{2}{*}{2} & \multirow{2}{*}{ Cabeza larga } & AF2613 & 3 & 2 & \begin{tabular}{l|l}
3 & 1 \\
\end{tabular} & $33-40$ \\
\hline & & AF5954 & 3 & 2 & \begin{tabular}{|l|l|}
2 & 0 \\
\end{tabular} & $33-39$ \\
\hline \multirow{2}{*}{2} & \multirow{2}{*}{ Necrópolis } & AF6797 & 3 & 3 & \begin{tabular}{|l|l|}
3 & 2
\end{tabular} & $36-43$ \\
\hline & & AF6830 & 3 & 1 & \begin{tabular}{l|l}
0 & 0 \\
\end{tabular} & $26-30$ \\
\hline \multirow{2}{*}{2} & \multirow{2}{*}{$\begin{array}{l}\text { Natchez } \\
\text { momia }\end{array}$} & AF6823 & 3 & 1 & \begin{tabular}{l|l}
0 & 0 \\
\end{tabular} & $26-30$ \\
\hline & & 108 & 3 & 1 & 100 & $29-33$ \\
\hline 1 & Nazca & AF1648 & 3 & 2 & \begin{tabular}{|l|l|}
2 & 2 \\
\end{tabular} & $33-43$ \\
\hline
\end{tabular}

Suturas: 1. Sutura incisiva, 2.sutura transversal palatina, 3. Sutura palatina media posterior, 4. Sutura palatina media anterior. Grados de cierre sutural: $0=$ sutura abierta, $1=$ cierre mínimo $(50 \%), 2$ = cierre significativo $(80 \%), 3$ = Obliteración Completa (100\%)

La edad de los cráneos con deformación tipo Opa (AF1643), Necrópolis (AF6797) fueron de 36-40 años, seguidos de los cráneos tipo Nazca (AF1648), Cabeza Larga (AF2613, AF5954) y Fronto-occipital (AF4885), (Fig.6) que tuvieron 33-43 ańos.

Un cráneo Aimara (AF10870), 1 Natchez (momia 108) tuvieron 29-33 años. Un cráneo Inca Costeño (AF0767), 1 Huaura (AF1699), 1 Necrópolis (AF6830) y un Natchez (AF6823), (Fig.8) estimaron 26-30 años. 
Un Opa (AF1577), 1 Cavernas (AF1741), 1 Huaura (AF4820), (Fig.10) estimaron entre 20-33 años; por último, 1 Frontooccipital (AF6025) tuvo 18-20 años y 1 Aimara (AF1489) menor de 18 ańos

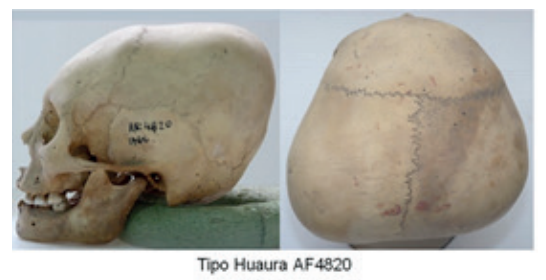

Fig 10 : Cráneo precolombino con deformación cefálica tipo Huaura, se aprecia obliteración sutural grado 1 por norma superior.

- Otros indicadores etarios presentes en la Cronología de Erupción Dentaria

Tabla 4: Relación lineal de resultados de estimación etaria de cráneos con deformación cefálica artificial

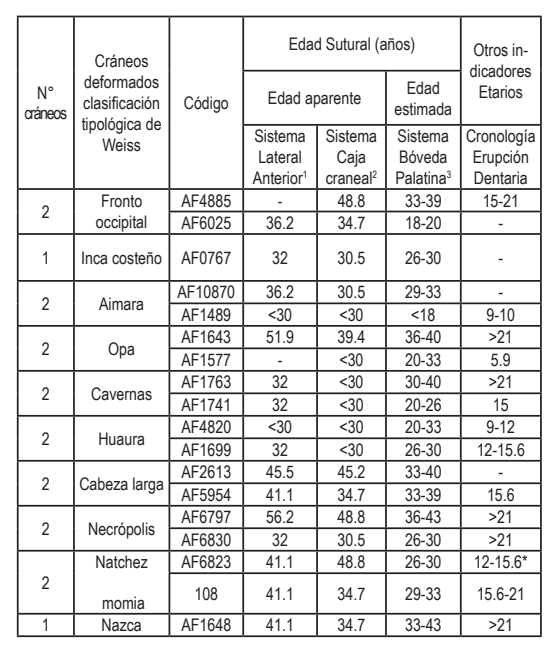

*Aparentemente canino deciduo persistente por ausencia de reemplazo

1,2: Método de Meindl \& Lovejoy

3. Método de Mann

Un cráneo con deformación tipo Opa (AF1643), 1 Cavernas (AF1763), $1 \mathrm{Ne}-$ crópolis (AF6797) y 1 Nazca (AF1648) estimaron tener más de 21 años.

Un Natchez (momia 108), 1 Cabeza Larga (AF5954), 1 Cavernas (AF1741) y 1 Fronto -occipital (AF4885), (Fig.6) estimaron entre 15-21 ańos. Un Huaura (AF1699) y 1 Natchez (AF6823), (Fig.8) tuvieron entre 12-15.6 años. Un Huaura (AF4820), (Fig.10) y 1 Aimara (AF1489) tuvieron entre 9-12 años.

Solo 1 cráneo Opa (AF1577) estimo tener 5.9 ańos. Los cráneos tipo Fronto - occipítal (AF6025), Inca Costeño (AF0767), Aimara (AF10870) y Cabeza Larga (AF2613) no presentaron dientes

\section{Discusión}

Las suturas craneanas, por sí solo, carecen de potencial de crecimiento innato y que el crecimiento sutural responden a influencias externas, de manera que postulamos como otros investigadores que los artefactos deformadores podrían haber modificado la permeabilidad y acelerado la obliteración de las suturas craneanas (Byron CD y Col2004"; Galvez y Col-20146)

La obliteración completa de las suturas del cráneo ocurre normalmente hasta una edad avanzada en el cráneo humano; sin embargo cráneos con deformación cefálica artificial, influenciados por factores ambientales, las suturas de la bóveda craneal se han visto obliterados entre los 15 y 21 ańos de edad dental, arrojando 48.8 ańos de edad sutural; discrepando con los mencionados por Sperber(1989) ${ }^{11}$ y Meike $(2002)^{12}$ en el sentido de que las suturas sagital, coronal y lambdoidea se consolida entre los 22 y 35 años

Encontramos coherencia entre los valores obtenidos en el punto pterion de los cráneos Necrópolis (AF6797) y Opa (AF1643) con los sugeridos por Meindl y Lovejoy ${ }^{19}$, respecto a que el pterion es un buen indicador de la década de los 40. Ademán observamos que el obelion tuvo actividad de cierre antes que el pterion (Figs.7,9,11)

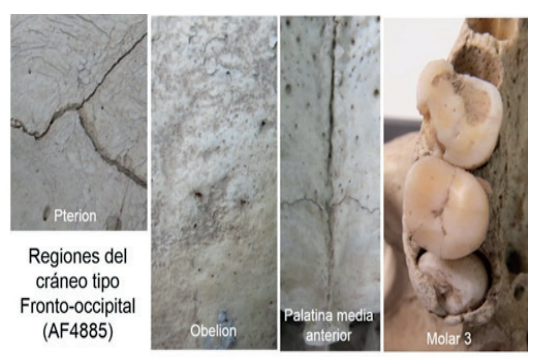

fig 7. Indicadores suturales y otros presentes en la cronología de erupción dentaria del cráneo precolombino con deformación cefálica artificial tipo Fronto-occipital

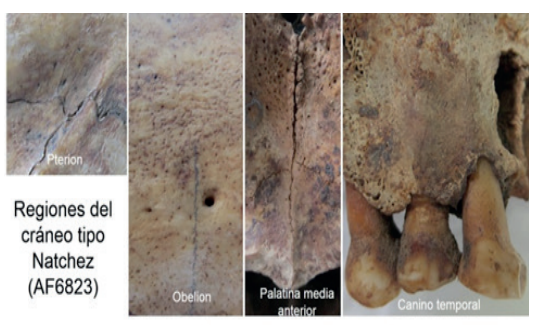

Fig.9: Indicadores suturales y otros presentes en la cronología de erupción dentaria del cráneo precolombino con deformación cefálica artificial tipo Natchez

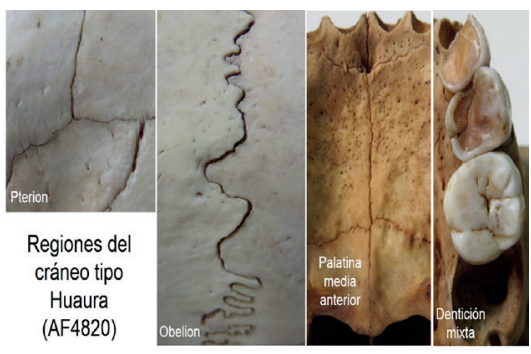

Fig.11: Indicadores suturales y otros presentes en la cronología de erupción dentaria del cráneo precolombino con deformación cefálica artificial tipo Huaura.

De los 18 cráneos con deformación cefálica artificial examinados ningún cráneo presento obliteración completa de las suturas palatinas, toda vez que sus edades fluctuaron entre 18 a 43 años; coincidiendo con las conclusiones de López Palafox ${ }^{23}$ en el sentido de que a los 50 años todas las suturas están cerradas.

Del mismo modo, un cráneo aimara de 9-10 ańos, valorado por indicadores de erupción dentaria, presentó signos de sutura incisiva abierta, lógicamente menor a 18 años en concordancia con López Palafox ${ }^{23}$

Las edades estimadas por los grados de obliteración de las suturas palatinas obtenidos en nuestro estudio (Tabla 3), caen dentro de la secuencia de obliteración sugerida por Navarro y Roldan ${ }^{24}$

Los indicadores etarios suturales comprometidos bajo la acción de los aperos deformadores expresaron valores altos, posiblemente aparentes; respecto a los valores bajos expresados por indicadores etarios suturales palatinos; los que se encontraron ajenos o alejados de la influencia de los citados aperos.

Los resultados obtenidos por los métodos de Meindl y Lovejoy, Mann y otros indicadores presentes en la cronología de erupción dentaria fueron ampliamente discrepantes en 15-20 años, vistos en una relación lineal entre ellos (Tabla 4)

\section{Conclusiones}

1. La edad estimada de los cráneos con deformación cefálica artificial por obliteración de las suturas de la caja craneal y lateral anterior, según el método de Meindl y Lovejoy ${ }^{19}$, expresó valores altos respecto a los obtenidos por cierre de las suturas palatinas, considerándose en este estudio como la edad aparente. 
2. La edad estimada de los cráneos con deformación cefálica artificial por obliteración de las suturas palatinas, según el método de Mann ${ }^{21,22}$, expresó valores bajos respecto a los obtenidos por cierre de las suturas craneanas; consideradas como la edad más aproximada.

3. La edad estimada de los cráneos con deformación cefálica artificial tomando en cuenta la cronología de erupción dentaria expreso valores bajos respecto a los obtenidos por los métodos de Meindl y Lovejoy $^{19}$ y Mann ${ }^{21,22}$ juntos.

4. La edad aparente y la edad estima$\mathrm{da}$ por los métodos establecidos fueron ampliamente discrepantes, siendo mayor la edad aparente superando a la estimada en 5 a 10 años. incrementándose aún más en $15 \pm 5$ años adicionales con los indicadores cronológicos de erupción dentaria.

\section{Agradecimiento}

Nuestro sincero agradecimiento al $\mathrm{Mu}$ seo Nacional de Arqueología, Antropología e Historia del Perú, por las facilidades técnicas y administrativas que hicieron posible el logro de los objetivos del presente estudio

\section{Referencias bibliográficas:}

1. Imbelloni J. Los pueblos deformadores de los Andes. La deformación intencional de la cabeza como arte y como elemento diagnóstico de las culturas. Anales del Museo Nacional de Historia Natural Bemardino Rivadavia, t. XXXVII, Publicación No 75. Buenos Aires, 1933.p.

2. Stewart, T.D. Skletal remains with cultural associations from Chicama, Moche and Viry valleys, Peru. Proc. U.S. Nat. Mus. 1943; 93:153-185.

3. Weiss P. Osteología Cultural, 2da. Parte: Tipología de las deformaciones cefálicas - estudio cultural de los tipos cefálicos y de algunas enfermedades óseas. Universidad $\mathrm{Na}$ cional Mayor de San Marcos, Lima 1960: 51-82.
4. Kauffmann DF. Manual de arqueología peruana. Lima: ediciones Peisa; 1973.336p.

5. Munizaga JR. Deformación craneana intencional en América. Rev Chil Antropol 1987(6):113-147

6. Gálvez, CL, Maita VL, Guillén BC, Menéndez ML. Implicancias culturales en la morfología craneana de grupos étnicos pre-colombinos peruanos. 2014;17(1):20-25

7. Torres-Rouff, C. La deformación craneana en San Pedro de Atacama. Estudios Atacameńos: Arqueología y Antropología Surandinas. 2007;33:25-38

8. Morriss-Kay GM, Wilkie AOM. Growth of the normal skull vault and its alteration in craniosynostosis: insights from human genetics and experimental studies. Journal of Anatomy 2005; 207: 637-653.

9. Enlow DH, Hans MG. Essentials of Facial Growth. W.B.United States of America: Saunders Company; 1996

10. Opperman LA. Cranial sutures as intramembranous bone growth sites. Developmental Dynamics 2000; 219: 472-485.

11. Sperber GH. Craniofacial Embriology. 4th edition. Wright, 1989.246p

12. Meike MC. Craniofacial Development, Growth and Evolution. edition. Bateson Publishing, Bressingham, Norfolk, England, 2002.363p.

13. Enlow DH. The human face: an account of the postnatal growth and development of the craniofacial skeleton. New York: Hoeber Medical Division. Harper \& Row publishers; 1968

14. Moore KL, Dalley AF. Anatomía con orientación clínica. $4^{\mathrm{a}}$ ed. Madrid: Editorial Médica Panamericana; 1996.

15. Griesemer DA, Sobczak JC.: Craniosynostosis. MedLink Neurology [CD-ROM] 2002.

16. Kabbani H, Raghuveer TS.: Craneosinostosis. American Familiy Phy- sician. 2004 Jun 15;69(12)28632870.

17. Byron CD, Borke J, Yu J, Pashley D, Wingard CJ, Hamrick M.: Effects of increased muscle mass on mousse saggital suture morphology and mechanics. Anat Rec A Discov Moll Cell Evol Biol. 2004 Jul;279(1):676-84.

18. Rouge D, Telmon N, Larrouy G, Duday H, Crubezy E, Arbus L. Comparaison des critéres visuels $\mathrm{du}$ pubis lors de la determination de $\mathrm{F}$ age des inidvidus au moment de la mort. Paris, Actes du colloque du C.E.R.S.A.M.S. Xéme Anniversaire, 1992, pp. 359-63.

19. Meindl RS, Lovejoy CO. Ectocranial suture closure: a revised method for the determination of skeletal age at death based on the lateral-anterior sutures. Am J Phys Anthropol 1985 Sep; 68(1):57-66.

20. Ramos A. M. Estimación de edad a través del grado de obliteración de las suturas craneales. Aplicabilidad del método Meindl y Lovejoy en Bogotá. Bogotá, Carrera de Antropología, Universidad de los Andes, 2003

21. Mann RW, Symes SA \& Bass WM. Maxillary suture obliteration: Ageing the human skeleton based on intact or fragmentary maxilla. Journal of Forensic Sciences. 1987; 32(1): 148-157

22. Mann RW, Jantz RL, Bass WM, Willey PS. J. Forensic Sci. Maxillary Suture Obliteration: A visual method for estimating skeletal age. Journal Forensic Sci. May,1991; 36(3): 781-91.

23. López Palafox J . Procedimientos Odontológicos en la investigación de un Genocidio -Ruanda. Madrid: Facultad de Odontología de la Universidad Alfonso X; 1994.

24. Navarro A, Roldán N. Método de Mann para estimar la edad esquelética en cráneos del Laboratorio de Criminalística de la Policía Nacional del Perú, octubre 2003 a julio 2004. Kiru 2007;4(1):2-7.

25. De Schour I, Massier M. The development of the human dentition. J Am Dent Assoc, 1941; 28:1153 